\title{
Nanoparticles and Their Toxicology Studies: A Green Chemistry Approach
}

\author{
Narendhran S* and Reshma K Nair \\ Department of Biotechnology, India \\ *Corresponding author: Narendhran S, Department of Biotechnology, Nava India Bus Stop, Avinashi Road, Coimbatore 641006, India

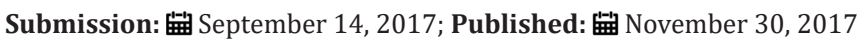

\begin{abstract}
Today the significance of nanoparticles have wide applicable in all fields. The misuse of various plant materials for the biosynthesis of nanoparticles considered a green innovation since it doesn't include any harmful chemicals. Nano particles with controlled size and synthesis are of principal and mechanical enthusiasm as they give answers for innovative and natural difficulties in the regions of catalysis, medicine, solar energy conversion and water treatment. The present review study manages the green union of metal (silver and gold) and metal oxide $\left(\mathrm{Fe}_{3} \mathrm{O}_{4}\right.$ and $\left.\mathrm{ZnO}\right)$ nanoparticles from marine sea weed plants and their vital applications in different fields and furthermore their toxicological studies and the significance of inclining towards the ocean weed plants and their characterization.
\end{abstract}

\section{Introduction}

Nanotechnology implies the creation of nanoparticles in size, shape, chemical composition and controlled diversity and potential uses for human benefits [1]. Nanoparticles are produced by chemical and physical methods are expensive, non-reliable and potentially dangerous to the environment. Alternatively nanoparticles produced by biological methods seem to gain interest as they are supposed to be inexpensive and eco-friendly. Biosynthesis of nanoparticles is a kind of bottom up approach and mainly by oxidation and reduction technique [2]. Nanotechnology has a wide range of applications mainly in the field of biomedical applications, drug delivery system, ultra- sensitive disease detection, etc. [3]. Nano sciences a new inter disciplinary subject that depends on the fundamental properties of nano size objects [4]. Nano particle possess wondrous optical, electronic, magnetic, and catalytic properties than the bulk material owing to their high surface area to volume ratio [5].

In recent areas a convergence between biological based technologies, green chemistry, and nanotechnology has taken place. The objective of this convergence is to create new materials and manufacturing processes that reduce or eliminate the use of hazardous substances [6]. An important aspect of nanotechnology is the synthesis of nanometer scale materials and the direct control of particle morphology and dimensions during formation. Nanometer scale materials at least one dimension less than $100 \mathrm{~nm}$ and can have a wide variety of geometric shapes such as plates, sheets, tubes, wires, and particles. Numerous studies have shown that nanometer scale material exhibit unique chemical, physical, electronic, optical, thermal, mechanical, and biological properties that significantly differ from their bulk scale counter parts [7]. These unique properties result from the extremely small size, shape, and size distribution. In addition, because of their small size, nano materials can act as bridge between bulk scale materials and molecular structure [8]. In terms of composition, nanomaterial can be broadly classified in to two types namely organic and inorganic. Organic nano materials are carbon based, while inorganic nano materials include noble metals (gold, silver), magnetic materials (iron oxide $\mathrm{Fe}_{3} \mathrm{O}_{4}$ ), and semiconductors such as titanium dioxide and zinc oxide.

The manufacture of nanoparticles can be broadly defined in to two approaches. The first is the top down approach and involves a material undergoing significant size reduction via physical or chemical processes $[9,10]$. During size reduction, the resulting particle size, shape, and surface structure are heavily dependent on the technique used. Unfortunately, size reduction also tends to introduce surface imperfections that can significantly impact the overall physicochemical properties of the fabricated nano particle. The second, bottom up approach builds nanoparticles via the assembly of atoms, molecules, and smaller particles or monomers [11]. Unfortunately, many of the chemical and physical processes used in both approaches suffer from several drawbacks such as low material conversion rates, are technically complex, have high energy requirements, and are generally expensive. Furthermore, many of these processes employ hazardous chemicals such as reducing agents, organic solvents, and non-biodegradable stabilizing agents. For examples, processes such as chemical precipitation and 
hydrolysis often result in toxic chemical species being deposited on the surface of newly formed nanoparticles. The presence of surface contaminants makes these nanoparticles unsuitable for clinical and biomedical applications [12]. Because of the drawbacks associated with conversional manufacturing processes there has been a growing interest in developing new ecofreindly production technologies based on the principles of green chemistry [13]. According, research in recent years has focused on manufacturing nano materials via nanotechnology- based processes that promote the principles green chemistry and reduce or totally and eliminate the use of hazardous chemicals .

Thus, ecofreindly green nanotechnology-based processes for the manufacture of nanoparticles have attracted considerable interest worldwide [14]. To emphasis this alternative approach, recent research has focused on using biological entities to synthesize a wide variety of nanoparticles. Biosynthesis via unicellular and multi cellular biological entities such as actinomycetes, bacteria, fungus, marine algae, plants, viruses, and yeast offer alternative ecofreindly approaches for producing nanoparticles. Each of these biological entities, to varying degrees, can perform as natural bio factories for producing particular nanoparticles.

Each of the biological entities has active molecules and compounds that can act as reducing agents and stabilizing agents to synthesize nanoparticles with diverse sizes, shapes, compositions and physicochemical properties [15]. The biologically diverse marine environment covers around $70 \%$ of the earth surface and is largely unexplored. Recent studies have shown that several marine plants have the ability to perform as bio factories for the production of nanoparticles [16]. In particular, interest in producing new and effective medicines from natural marine plant sources has revealed that several forms of marine algae [17] have the potential to produce a variety of nanoparticles. A number of these nanoparticles types have been found to be effective antimicrobial agents [18]. Marine algae contain a diverse range of different species, which are generally classified in to two groups, namely, microalgae and macroalgae. Microalgae species such as phytoplankton survive suspended in the water column, while macroalgae (commonly known as seaweed) are plant-like organisms that can range in size from a few centimeters up to several meters in length. For example, the giant kelp grows up from the seafloor to form vast under water forests. Seaweeds have adapted to living in a variety of habitat, ranging from small tidal rock pools close to shore or living several kilometers offshore in seawater depths capable of receiving sufficient light to promote photosynthesis. Algae are broadly classified into three groups based on the algal body or thallus pigmentation. The color groups are brown algae (phaeophytes), green algae (chlorophytes), and redalgae (rhodophytes) [19]. Brown and red algae are predominantly marine based, with some species of red algae being found in water depths where light levels are extremely low, while green algae is found in both marine and fresh water environments.

The oceans provide unlimited space for capturing solar energy by marine plants through photosynthesis. Marine plants comprise of algae, sea grasses, mangroves and sand dune vegetation. The microscopic algae, is known as phytoplankton and macroscopic ones as seaweeds. Most people come in contact with seaweeds that are washed ashore by the incoming tides [20]. Seaweeds are marine macro algae and primitive type of plants, growing abundantly in the shallow waters of sea, estuaries and back waters. They flourish wherever rocky, coral or suitable substrata are available for their attachment. Seaweeds are one of the most important and abundantly available marine living resources. They are not fully exploited and underutilized. Seaweeds are classified into three groups' viz. green algae, brown algae and red algae and each of these groups differs with regard to their reserve food material and cell-wall polysaccharides.

Seaweeds grow in the intertidal as well as in the sub tidal area up to a certain depth where $0.1 \%$ photosynthetic light is available. Seaweeds are the first organism in the marine food chain, which provide nutrients and energy for other living organisms. They are one of the ecologically and economically important living resources of the world's oceans. Being the oldest family of plants on earth, they have admirable qualities of being flexible, tenacious and prolific. Seaweeds provide shelter and habitat for many coastal animals. Seaweeds have many direct uses, traditionally consumed in different parts of the world. Recently human consumption of green algae (5\%), brown algae (66.5\%) and red algae $33 \%$ ) is higher in Asia, mainly in Japan, China and Korea. In Asian countries, seaweeds are often consumed as marine vegetables. Seaweeds are major coastal resources which have been utilized for the extraction of phycocolloids as alginates from brown algae, agar and carrageen an from red algae. The major stress on seaweeds in the coastal zone of Tamil Nadu is pollution, through various means. 90 species of seaweeds belonging to chlorophyceae, phaeophyceae and rhodophyceae were identified from Hare Island of Tuticorin. Sreekaladeviet also studied the distribution of marine macro algae in Idinthakarai and Vizhinjam coasts.

\section{Types of nanoparticles}

Nanoparticles are characterized in to real sort's viz. natural and inorganic nanoparticles. Carbon nanoparticles are known as natural nanoparticles. Magnetic nanoparticles, noble metal nanoparticles (platinum, gold and silver) and semiconductor Nanoparticles (titanium dioxide and zinc oxide) are gathered as inorganic nanoparticles. Inorganic nanoparticles are progressively utilized as used in drug delivery due to their distinctive features such as ease of use, good functionality, biocompatibility, ability to targeted specific cell and controlled release of drugs.

There are 3 methods for preparation of nanoparticles.

a. Physical

b. Chemical

c. Biological

Traditionally nanoparticles were produced only by physical and chemical methods. Some of the common methods are ion 
sputtering, solvothermal synthesis, reduction and sol gel technique. The need for biosynthesis of nanoparticles rose as the physical and chemical processes were cost effective. Nature has devised various processes for the synthesis of nano and micro length scaled inorganic materials which have contributed to the development of relatively new and largely unexplored area of research based on the biosynthesis of nanomaterials. Hence, for the biosynthesis of nanoparticles plant and microbial sources were used.

\section{Green synthesis of nanoparticles}

The green method of synthesis of nanoparticles is easy, efficient, and eco-friendly in comparison to chemical-mediated synthesis [20]. The chemical synthesis involves toxic solvents, high pressure, energy and high temperature conversion and microbe involved synthesis is not feasible industrially due to its lab maintenance. Since, green synthesis is the best option to opt for the synthesis of nanoparticles. Use of chemical and physical method in the synthesis of nanoparticles is very expensive and cumbersome. The chemical and physical methods of nano particle synthesis lead to the presence of some toxic chemicals absorbed on the surface that may have adverse effects in applications, so there is a growing need to develop environmentally benign nanoparticles. Researchers have used biological extracts for the synthesis of nanoparticles, by adopting simple protocols, involving in the process of reduction of metal ions by using biological extracts as a source of reductants either extracellular or intracellular. Synthesis of nanoparticles may be triggered by several compound such as carbonyl groups, terpenoids, phenolics, flavonones, amines, amides, proteins, pigments, alkaloids and other reducing agents present in the plant extracts and microbial cells [21,22]. The exact mechanism of nanoparticles synthesis by biological extracts is yet to be understood (Figure 1).

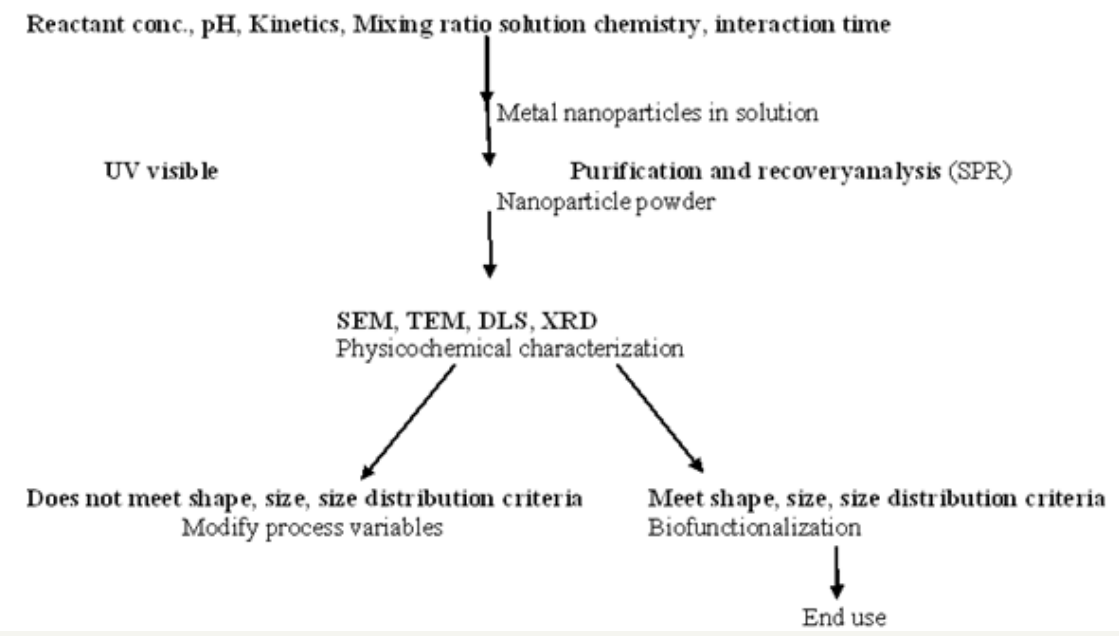

Figure 1: General methodology of nanoparticle synthesis.

Three types of green synthesis:

a. Use of microorganisms like fungi, yeasts (eukaryotes) or bacteria, actinomycetes (prokryotes),

b. Use of plant extracts or enzymes

c. Use of templates like DNA, membranes, viruses and diatoms. Bio-reductant from bacteria, fungi, or plant parts + Metal ions (Maybe enzyme/ phytochemical).

\section{Characterization of nanoparticles}

Nanoparticles can be characterized by their shape, size, surface area and disparity [23]. For their characterization some common techniques are used like UV-visible spectrophotometer, dynamic light scattering (DLS), scanning electron microscopy (SEM), transmission electron microscopy (TEM), Fourier transforms infrared spectroscopy (FTIR), X-ray diffraction (XRD) \& energy dispersive spectroscopy (EDS) [24], auger electron spectroscopy (AES), X-ray photoelectron spectroscopy (XPS), time of flight secondary ion mass spectrometry (TOF-SIMS), low energy ion scattering (LEIS), scanning tunneling microscopy (STM) and atomic force microscopy (AFM), scanning probe electron microscopy (SPM) etc. UV-Vis spectrophotometer allows identification, characterization and analysis of metallic nanoparticles. Generally $300-800 \mathrm{~nm}$ light wavelength is used for the characterization of size range 2 to $100 \mathrm{~nm}$ [25]. The dynamic light scattering (DLS) is used to characterize the surface charge \& the size distribution of nanoparticles. Electron microscopy is a common method for surface and morphological characterization. Scanning electron microscopy (SEM) \& transmission electron microscopy (TEM) are used for the morphological characterization at the nanometer to micrometer scale. SEM can provide morphological information on the submicron scale \& elemental information at the micron scale, but TEM has a 1000 fold higher resolution compared with the SEM. Characterization of nano particle using FTIR is very useful for the surface chemistry because the organic functional groups can be determined which are attached to the surface of nanoparticles. XRD is used to examine the overall oxidation state of the particles as a function of time, i.e. phase identification \& characterization of the crystal structure of the nanoparticles [24] to know the elemental composition of metal nanoparticles EDS is used [25]. AES \& XPS, 
TOFSIMS, scanning probe microscopy (SPM) techniques are important for the primary surface analysis of nanoparticles.

AES and XPS are used to determine the presence, composition $\&$ thickness of coating on nanoparticles, surface enrichment and depletion at particle surfaces. Sometimes XPS is used to determine particle sizes when conditions are not appropriate for analysis by other methods. TOF-SIMS is useful for obtaining molecular information about surface layers, functional groups which are added to the surface. In LEIS process the amount of energy lost by the ion during this scattering process due to a low energy ion beam can be determined. This scattering process is used to determine the identity of the elements present in the outermost surface of the material under analysis. Recently it is found that it is useful due to its high sensitivity to the outermost atomic layers of a sample [26]. AFM and STM provide surface characterization at the atomic scale.

\section{Toxicology of Nanoparticles}

Nanoparticles with their unique size-dependent properties are at the forefront of advanced material engineering applications in several fields. However, recent concerns about the toxicological impact of nanoparticles to human health and the environment have created uneasiness in the wider community. Larger surface area to-volume ratios of nanoparticles mean that more parameters must be considered when compared to conventional bulk Scale materials. For example, parameters such as particle size, morphology, composition, concentration, chemical reactivity, dispersion, and aggregation can all directly influence the behavior and interactions between nanoparticles and specific environments. In the case of nano particle size, studies have shown that particles around $10 \mathrm{~nm}$ can induce greater human cell death rates when compared to larger particles ranging in size from 50 to $100 \mathrm{~nm}$ [27]. Furthermore, nanoparticles of the same composition can display behavioral differences when interacting with different environments. These differences can result from slight variations in particle size, morphology, surface reactivity, and surface coatings. Another source of behavioral differences can also result from manufacturing process. For example, conventional chemical and physical manufacturing processes tend to use hazardous chemicals such as surfactants. These surfactants act as sculpturing agents that direct particle growth. While capping agents are generally used to stabilize and prevent nanoparticles aggregation. Toxicity issues arise because it is extremely difficult to remove all chemical contaminants from the surface of nanoparticles [28]. Furthermore, studies have shown that naked nanoparticles do not exist for very long in biological or environmental surroundings. Their high surface energies attract atoms and molecules, which rapidly coat and change, surface properties [29]. In a biological setting, biomolecules such as enzymes and proteins rapidly attach to nanoparticles to form surface layering or corona. The difficulty in determining toxicity arises from how nanoparticles bind and interacts with biological materials and living organisms. How these binding and interaction mechanisms change nanoparticle surface properties and their subsequent influence in living organisms are not fully understood [30]. For example, silver ( $\mathrm{Ag}$ ) has been used as and to nano particle surface to form an equilibrium layer with the surrounding environment. Antimicrobial agent for centuries and the use of Ag nanoparticles in recent years has significantly increased the effectiveness of $\mathrm{Ag}$ as a medicinal preparation [31]. However, using high concentrations of Ag nanoparticles can induce toxicity and as a consequence produce a variety of health problems. Furthermore, studies have also shown the release of Ag nanoparticles into the environment can produce significant Ecological problems [32]. Therefore, toxicity results not only from nanoparticle size, morphology, composition, and surface reactivity, but also from the presence of surface contaminants. This added complication makes it necessary to understand the interaction of formation chemicals during synthesis and ultimately the interactions occurring in environment to determine potential toxicity issues [33]. To reduce the toxicity concerns, there needs to be greater attention paid to finding alternative clean, nontoxic, and eco-friendly green chemistry based approaches for manufacturing nanoparticles. Importantly, the interactions between a nanoparticle and its surrounding environment are a surface phenomenon [34]. Thus, surface and surface contaminants are actively involved in the nanoparticle/environment interface rather than the nanoparticles material core. Because of the importance of nanoparticle/ environmental interactions, the presence of any harmful surface toxic residues will prevent nanoparticles being used in therapeutic applications. Thus, the medical use of nanoparticle-based pharmaceuticals will be ultimately evaluated on their therapeutic benefit against any toxicity risks. Therefore, in an attempt to reduce toxicity risks, recent research has focused on biologically based synthesis techniques. Biosynthesis of nanoparticles via biological entities has the potential to deliver a clean and eco-friendly approach. In particular, biomolecules present in marine algae and sea grasses have the capability to synthesize wide variety of nanoparticles with very low to no toxicity risks.

\section{Metal Nanoparticles}

The biogenic synthesis of metal nanoparticles using marine algae from precursor metal salts is a facile room temperature process. Synthesis begins by mixing a metal salt solution of known concentration with an aqueous solution containing a seaweed extract. During the initial reduction period, there is a color change in the reaction mixture indicating that nanoparticle nucleation has taken place. With the passage of time the small neighboring nucleonic particles in the reaction mixture start agglomerating to form larger and more thermodynamically stable nano particles. The aggregation and self-assembly of the nucleonic particles are assisted by biomolecules present in the seaweed extract. During self-assembly, the most energetically favorable and stable particle shapes are formed. Typical shapes formed include cubes, hexagons, pentagons, rods, spheres, triangles, and wires. Studies have also shown that nanoparticle formation is influenced by several experimental factors.

These factors include

a. Algae concentration 
b. Metal salt concentration

c. Reaction time

d. Reaction solution $\mathrm{pH}$

e. Temperature

Controlling these factors will determine the quality, size, and shape of the biosynthesized nanoparticles. The vast majority of marine plant studies have examined the biogenic synthesis of Ag and Au nanoparticles using seaweeds.

\section{Silver (Ag) Nanoparticles}

Historically, Ag has attracted considerable interest due to its antimicrobial properties [35]. Advances in manufacturing processes have enabled the large scale production of Ag nanoparticles with unique physiochemical and antimicrobial properties. The antimicrobial properties and efficacy of Ag nanoparticles towards a variety of microbial entities have resulted in their incorporation into a wide range of pharmaceuticals and medical protocols [36]. The mechanisms associated with their antimicrobial properties are not fully understood, but the interaction between $\mathrm{Ag}$ nanoparticles and microbial cell membranes is believed to cause significant membrane damage and biosorption. The increased cellular uptake of nanometer scale Ag results in significant toxicological damage to cellular DNA [37]. From another perspective, seaweeds are a valuable source of bioactive materials that include a variety of polysaccharides. Due to the presence of these materials, seaweeds are considered to have medicinal and pharmaceutical properties. Therefore, using seaweeds to biosynthesize Ag nanoparticles has the potential to deliver an additional synergic effect and produce nanoparticles with enhanced medicinal properties. The biosynthesis of Ag nanoparticles using several species of seaweed has been reported by a small number of researchers in recent years. For example, Rajeshkumar et al. [37] have reported the biosynthesis of Ag nanoparticles using brown seaweed Padinatetrastromatica. Their study revealed that the nanoparticles were spherical in shape, had a mean particle size of $14 \mathrm{~nm}$, and displayed antibacterial activity [38]. Similarly, crystalline Ag nanoparticles were biosynthesized from Codiumcapitatum to produce spherical and cubic nanoparticles ranging in size from 3 to $44 \mathrm{~nm}$, with a mean particle size of $30 \mathrm{~nm}$. A comparable result was also reported for green alga Spirogyra insignis, which also produced spherical nanoparticles with a mean particle size of $30 \mathrm{~nm}$, while macroalga Padinatetrastromatica was found to produce crystalline spherical nanoparticles ranging in size from 5 to $35 \mathrm{~nm}$. Ag nanoparticles synthesized by seaweeds have also been found to have antifungal [39], antibacterial, and anticancer properties [40]. On another front, Ramkumar et al. have used anaqueous solution containing an extract taken from Turbinariaconoides to biosynthesize both Ag and Au nanoparticles. Their study also investigated the anti biological film formation activity of the nanoparticles against marine biofilm forming bacteria. Also, studies into antimicrobial agents have found that $\mathrm{AgCl}$ nanoparticles biosynthesized using an aqueous extract containing Sargassumplagiophyllum were effective agents against bacterial pathogens such as Escherichia coli [41].

\section{Gold (Au) nanoparticles}

$\mathrm{Au}$ nanoparticles with their unique size dependent properties have been used in a variety of applications that include catalytic agents, biosensors, pharmaceuticals, imaging, and electronics [42]. Because of their unique properties and applications, research has focused on using alternative methods for manufacturing $\mathrm{Au}$ nanoparticles. In recent years biological entities have been examined as potential biofactories. And as a result, the biologically diverse marine environment is starting to attract the interest of more researchers worldwide. Because of this interest, a number of recent articles describing the biosynthesis of $\mathrm{Au}$ nanoparticles by both marine and fresh water alga have appeared in the literature. A study by Romero-Gonz'alez et al. [43] has shown that dealginated seaweed waste can be used to reduce $\mathrm{Au}$ ions in solution to form Au particles ranging from the nanometer scale range up to around $6 \mu \mathrm{m}$ in size. Their study found the functional groups present in the seaweed were capable of producing Stable particles of the various shapes that include irregular, decahedral, and hexagonal rods and tetrahedral platelets [44]. In a similar study, Mata et al. were able to demonstrate an eco-friendly process that recovered $\mathrm{Au}$ from dilute hydrometallurgical solutions. The process involved the biosorption and bioreduction of Au by brown seaweed (Fucusvesiculosus) and resulted in nanoparticles of various sizes and morphologies being produced.Singaravelu Getal. [38] havealso reported the biosynthesis of Au nanoparticles using a marine alga Sargassumwightii Greville. In their study the alga rapidly produced stable nanoparticles ranging in size from 8 to $15 \mathrm{~nm}$ and was spherical in shape [45]. In a similar studies by Luangpipat et al. [46] (Chlorella vulgaris) , Rajasulochana et al. [47](Kappaphycusalvarezii) and Stalin Dhas et al. [42] (Sargassummyriocystum) were able to produce a wide range of stable nanoparticle sizes, while Senapati et al. [48] have reported the biosynthesis of $\mathrm{Au}$ nanoparticles using marine alga Tetraselmiskochinensis and Rajeshkumar et al. [37] have also reported using Turbinariaconoides to produce Au nanoparticles ranging in size from 6 to $10 \mathrm{~nm}$ and shapes that included spherical, pseudo spherical, and triangular shapes. In addition, Castro et al. have reported the biosynthesis of Au nanoparticles using green alga Spirogyra insignis and red alga Chondruscrispus [49]. And recently, Arockiya Aarthi Rajathi et al. [50] have reported the biosynthesis of Au nanoparticles using a brown alga Stoechospermummarginatum. Their study revealed the nanoparticles were crystalline and ranged in size from 18.7 to $93 \mathrm{~nm}$ and were predominantly spherical in shape with small numbers of hexagonal and triangular platelets. The study also found the hydroxyl groups of the diterpenoids present in the brown seaweed were directly involved in $\mathrm{Au}$ reduction. The nanoparticles also displayed significant antibacterial activity against a range of selected bacterial pathogens. Crystalline $\mathrm{Au}$ nanoparticles ranging in size from 7 to $1 \mathrm{~nm}$ have also been biosynthesized using brown seaweed (Turbinaria ornate) and by another brown seaweed (Padinapavonica) to produce spherical nanoparticles ranging in size from 30 to70nm [51]. Additionally, studies have shown that two species of freshwater algae [green alga (Prasiolacrispa) and red alga (Lemaneafluviatilis)] are capable of biosynthesizing $\mathrm{Au}$ nanoparticles [52]. Furthermore, 
current preliminary research by the authors has shown that an aqueous solution containing seagrass rhizome extract can biosynthesize Au nanoparticles at room temperature. The seagrass (Posidoniaaustralis) is a marine flowering plant that has adapted to living in the near shore environment along the southern waters of temperate Australia. During biosynthesis the reactive mixture changed color from a light yellow to a dark brown, which indicated the formation of Au nanoparticles.

\section{Metal Oxide Nanoparticles}

Metal oxides are an interesting class of inorganic materials that have been extensively explored and studied due to their wide range of structures and properties. The character of metal oxides is more complex than pure metals, with metal oxide bonding varying from nearly ionic to highly covalent and even metallic in nature. Metal oxides come in a variety of different forms, each possessing unique compositions, morphologies, structures, and physiochemical properties [53]. In particular, metal oxide nanoparticles are of particular interest due to their unique and phenomenal optical, electronic, and magnetic properties. These unique properties give metal oxide nanoparticles significant industrial importance in a variety of applications that include catalytic processes, electronics, sensors, magnetic storage media, and solar energy conversion. Current literature describing the biosynthesis ofmetal oxide nanoparticles via marine algae is very limited and has mainly focused on three metal oxide types, namely, copper oxide, ferric oxide, and zinc oxide. Abboud et al. [54] have reported the biosynthesis of copper oxide nanoparticles using a brown alga extract (Bifurcariabifurcata). The facile process produced both cuprous oxide nanoparticles $\left(\mathrm{Cu}_{2} \mathrm{O}\right)$ and cupric oxide nanoparticles (CuO). The majority of the nanoparticles were spherical in shape, with a small percentage being slightly elongated. The nanoparticles ranged in size from $5 \mathrm{~nm}$ to $45 \mathrm{~nm}$ and were found to have a mean particle size of $22.6 \mathrm{~nm}$. Subsequent antibacterial studies using Enterobacteraerogenes and Staphylococcusaureus revealed that the copper oxide nanoparticles had good antibacterial properties against both bacterial species. A recent study by Khanehzaei et al. [55] has also reported the biosynthesis of copper cored copper oxide nanoparticles using red seaweed (Kappaphycusalvarezii) extracts. The seaweed acted as an effective stabilizing agent and produced copper cored-cuprous oxide coated nanoparticles that were spherical in shape with a mean particle size of $53 \mathrm{~nm}$. Their study also found the nano particle surfaces were capped bypaired oxygen atoms, some hydroxyl and sulphates groups from the watersoluble sulphated polysaccharides derived from the cell walls making up the seaweed [56].

A recent study by Mahdavi et al. [17] found that ferric oxide $\left(\mathrm{Fe}_{3} \mathrm{O}_{4}\right)$ nanoparticles could be synthesized by one-step green biogenic method using brown seaweed (Sargassummuticum). The aqueous seaweed extract was mixed with an aqueous ferric chloride solution to produce $\mathrm{Fe}_{3} \mathrm{O}_{4}$ nanoparticles. The amino, carboxyl, and hydroxyl functional groups derived from the watersoluble polysaccharide cell walls were found to act as both reducing agent and capping agent. The mean particle size produced was
$18 \pm 4 \mathrm{~nm}$, crystalline in nature, and having a cubic morphology [57]. Subsequent in vitro studies by Names and confirmed their potential use in the treatment of cancer.

Current biosynthesis research using seaweeds has also examined protocols for producing zinc oxide ( $\mathrm{ZnO}$ ) nanoparticles. ZnO nanoparticles have exceptional electrical and optical properties, which makes them suitable for a wide range of applications such as biomedical, photo catalysts and solar cells [58]. Recently Nagarajan \& Kuppusamy et al. [53] examined three types of seaweed that included green (Caulerpapeltata), red (Hypneavalentiae), and brown (Sargassummyriocystum) for the biosynthesis of $\mathrm{ZnO}$ nanoparticles. After investigating experimental parameters such as metal ion concentration, seaweed extract concentration, temperature, $\mathrm{pH}$, and reaction time, brown seaweed (Sargassummyriocystum) was found to be effective in producing $\mathrm{ZnO}$ nanoparticles [58]. Their study also revealed that the soluble photo chemicals present in Sargassummyriocystum such as alginic acid, ascorbic acid, protein, carbohydrates, flavanoids, tannins, mannitols, and lipids acted as both reduction and stabilizing agents. The morphology of the synthesized nanoparticles included spherical, triangular, hexagonal, rod, and rectangular types. The particle sizes ranged from $76 \mathrm{~nm}$ up 186nm. Furthermore, both particle size and morphology were found to be strongly influenced by the experimental parameters. For example, both particle size and morphology were influenced by temperature, while biosynthesis carried out at $\mathrm{pH} 8$ tended to predominantly produce nanoparticles with a mean size of $36 \mathrm{~nm}$.

\section{Applications of Nanoparticles}

Nanoparticles have pulled in impressive enthusiasm for late years and as needs have been broadly announced in the 10 diary of nano science literatures.The one of a kind sizeand shape subordinate physic chemical properties make nano materials more intuitive and responsive to certain compound species contrasted with their bulk scale partners. The novel properties have been extensively investigated and evaluated for a wide range of applications in a number of fields. Among noble metals, Ag nanoparticles are used as a broad spectrum antimicrobial agent in the biomedical field. The biosynthesis of $\mathrm{Ag}$ and $\mathrm{Au}$ nanoparticles by marine algae has focused on their antimicrobial properties against several pathogens. Therefore, future studies are needed to confirm that the physiochemical properties of Ag and Au nanoparticles synthesized by marine algae have similar properties to those produce by more conventional manufacturing processes and can be used in other applications. This is of particular importance since conventionally manufactured $\mathrm{Au}$ nanoparticles have been used in a variety of biomedical applications such as carriers for anticancer drugs [59], biosensors [60], and targets in tumors for thermal treatment therapies [61]. $\mathrm{Fe}_{3} \mathrm{O}_{4}$ nanoparticles are another interesting type of nanometer scale material with unique super paramagnetic properties. The magnetic properties of the magnetite nanoparticles make them ideal candidates for magnetic resonance imaging (MRI) and targeted drug delivery applications [62]. However, very few studies have reported using marine algae to produce $\mathrm{Fe}_{3} \mathrm{O}_{4}$ nanoparticles similarly; very few studies have been reported in 
the literature describing the biosynthesis of zinc oxide and copper oxides via marine algae. Oxides are of particular interest, since copper oxide nanoparticles have antibacterial properties and zinc oxide nanoparticles display both therapeutic and photo catalytic properties [62]. Overall, the biogenic synthesis of metal and metal oxide nanoparticles using marine algae and marine plants is a largely unknown area of research with relatively few articles being reported in the literature. This review has summarized the currently available articles reported in the literature. However, the relatively small number of articles appearing in the literature indicates that further research is needed to fully explore the prospective properties of marine algae and marine plants for the biosynthesis of metal and metal oxide nanoparticles. In addition, further research is needed to fully explore the potential use of these metal and metal oxide nanoparticles in new pharmaceuticals and medical treatments.

\section{Conclusion}

The biogenic amalgamation of metal and metal oxide nanoparticles has pulled in significant enthusiasm for late years. This intrigue springs from one of kind nano particle properties, which makes them profoundly alluring for an extensive variety of pharmaceutical and biomedical applications. The biogenic combination of nanoparticles by marine ocean weeds can possibly convey an easy green, and eco accommodating methodology can possibly convey on option way to deal with traditional assembling process that tend to utilize poisonous chemicals and solvents. The examinations talked about in the present audit have revealed that polysaccharides, proteins and other bioactive chemicals found in the cell layer of marine ocean weeds act as both reducing and capping agents. In any case, a generally modest number of marine ocean weeds have been concentrates to date and examination range remains to great extent unknown. This there are novel open doors for investigation and growing new green based biogenic procedures for the generation of metal and metal oxide nanoparticles. Ideally this review will motivate quite needed examine in this generally new and uninvestigated field.

\section{References}

1. Giridharan T, Chandran MS, Sindhu S, Arumugam P (2014) Studies on green synthesis, characterization and anti-proliferative potential of silver nano particle using Dodonaea viscosa and Capparis deciduas. Biosci Biotechnol Res Asia 11(2): 665-673.

2. Curtis ASG, Wilkinson C (2001) Nano techniques and approaches in biotechnology. Trends Biotech 19(3): 97-101.

3. Wilkinson JM (2003) Nano technology applications in medicine. Med Device Technol 14(5): 29-31.

4. Abou El-Nour KMM, Eftaiha A, Al-Warthan A, Ammar RAA (2010) Synthesis and applications of silver nanoparticles. Arabian Journal of Chemistry 3(3): 135-140.

5. Poulose S, Panda T, Nair PP, Th'eodore T (2014) Bio synthesis of silver nanoparticles. Journal of Nano science and Nano technology 14(2): 2038-2049.

6. Anastas PT, Warner JC (2000) Green Chemistry: Theory and Practice, Oxford University Press, Oxford, UK.
7. Bogunia-Kubik K, Sugisaka M (2002) From molecular biology to nano technology and nano medicine. Bio Systems 65(2-3): 123-138.

8. Mansoori GA, George TF, Assoufid L, Zhang G (2007) Molecular building blocks for nanotechnology, In: Parsons JG, Peralta-Videa JR, GardeaTorresdey JL (Eds.), vol. 109 of Topicsin Applied Physics, Springer, New York, NY, USA.

9. Nielson R, Kaehr B, Shear JB (2009) Micro replication and design of biological architectures using dynamic-mask multiphoton lithography. Small 5(1): 120-125.

10. Birnbaum AJ, Pique A (2011) Laser induced extra-planar propulsion for three-dimensional micro fabrication. Applied Physics Letters 98(13): 134101-134106.

11. Poinern GEJ, Chapman P, Le X, Fawcett D (2013) Green biosynthesis of gold nanometre scale plates using the leaf extracts froman indigenous Australian plant Eucalyptusmacrocarpa. Gold Bulletin 46(3): 165-173.

12. Albrecht MA, Evans CW, Raston CL (2006) Green chemistry and the health implications of nanoparticles. Green Chemistry 8(5): 417-432.

13. Duan H, Wang D, Li Y (2015) Green chemistry for nanoparticle synthesis. Chemical Society Reviews 44(16): 5778-5792.

14. Kulkarni N, Muddapur U (2014) Biosynthesis of metal nanoparticles: a review. Journal of Nanotechnology.

15. Mohanpuria P, Rana NK, Yadav SK (2008) Biosynthesis of nanoparticles: technological concepts and future applications. Journal of Nanoparticle Research 10(3): 507-517.

16. Mohanpuria P, Rana NK, Yadav SK (2008) Biosynthesis of nanoparticles: technological concepts and future applications. J Nanopart 10: 507-517.

17. Mahdavi M, Namvar F, Bin Ahmad M, Mohamad R (2013) Green biosynthesis and characterization of magnetic iron oxide $\left(\mathrm{Fe}_{3} \mathrm{O}_{4}\right)$ nanoparticles using seaweed (sargassummuticum) aqueous extract. Molecules 18(5): 5954-5964.

18. Monalisa P, Nayak PL (2013) Eco friendly green synthesis of iron nanoparticles from various plants and spices extract. International Journal of Plant, Animal and Environmental Sciences 3(1): 68-78.

19. Mohanpuria P, Rana KN, Yadav SK (2008) Biosynthesis of nanoparticles: technological concepts and future applications. J Nanopart 10(3): 507517.

20. Leela A, Vivekanandan M (2008) Tapping the unexploited plant resources for the synthesis of silver nanoparticles. Afr J Biotechnol 7(17): 3162-3165.

21. Jiang J, Oberdorster G, Biswas P (2009) Characterization of size, surface charge and agglomeration state of nanoparticle dispersions for toxicological studies. Nanopart 11(1): 77-89.

22. Feldheim DL, Foss CA (2002) Metal nanoparticles: synthesis, characterization and applications. Boca Raton 124(26): 7874-7875.

23. Chitrani BD, Ghazani, AA, Chan WCW (2006) Determining the size and shape dependence of gold nanoparticle uptake into mammalian cells. Nano Lett 6(4): 662-668.

24. Sun S, Murray CB, Weller D, Folks L, Moser A (2000) Mono disperse FePt nanoparticles and ferromagnetic FePt nanocrystal superlattices. Science 287: 1989-1992.

25. Brongersma HH, Draxler M, Ridder De M, Bauer (2007) Surface composition analysis by low-energy ion scattering. Surface Science Reports 62(3): 63-109.

26. Gorth DJ, Rand DM, Webster TJ (2011) Silver nanoparticle toxicity in Drosophila: size does matter. International Journal of Nanomedicine 6 : 343-350.

27. Gautam A, Van Veggeln FCJM (2013) Synthesis of nanoparticles, their biocompatibility, and toxicity behavior for biomedical applications. 
Journal of Materials Chemistry B 1(39): 5186-5200.

28. Cedervall T, Lynch I, Lindman S, Berggård T, Thulin E, et al. (2006) Understanding the nanoparticle-protein corona using methods to quantify exchange rates and affinities of proteins for nanoparticles. Proceedings of the National Academy of Sciences of the United States of America 104(7): 2050-2055.

29. Nowack B (2009) The behavior and effects of nanoparticles in the environment. Environmental Pollution 157(4): 1063-1064.

30. Elsaesser, Howard CV (2012) Toxicology of nanoparticles. Advanced Drug Delivery Reviews 64(2): 129-137.

31. Prabhu S, Poulose E (2012) Silver nanoparticles: mechanism of antimicrobial action, synthesis, medical applications, andtoxicity effects. International Nano Letters 2(32): 1-10.

32. Choi O, Deng KK, Kim NJ, Ross L, Surampalli RY, et al. (2008) The inhibitory effects of silver nanoparticles, silver ions, and silver chloride colloids on microbial growth. Water Research 42(12): 3066-3074.

33. Lynch, Cedervall T, Lundqvist M, Cabaleiro-Lago, Linse S, et al. (2007) The nanoparticle-protein complex as a biological entity; a complex fluids and surface science challenge for the $21^{\text {st }}$ century. Advances in Colloid and Interface Science 134-135: 167-174.

34. Shah M, Fawcett D, Sharma S, Tripathy SK, Poinern GEJ (2015) Green synthesis of metallic nanoparticles via biological entities. Materials 8(11): 7278-7308.

35. Smit J (2004) Medicinal and pharmaceutical uses of seaweed natural products: a review. Journal of Applied Phycology 16(4): 245-262.

36. Princy F, Gopinath A (2015) Eco-friendly synthesis and characterization of silver nanoparticles using marine macroalga Padina tetrastromatica. International Journal of Science and Research 4(6): 1050-1054.

37. Rajeshkumar S, Kannan C, Amnadurai G (2012) Synthesis and characterization of antimicrobial silver nanoparticles using marine brown seaweed Padinatetrastromatica. Drug Invention Today 4(10): 511-513.

38. Govindaraju, Kiruthiga V, Kumar VG, Singaravelu G (2009) Extracellular synthesis of silver nanoparticles by a marine alga, Sargassumwightiigrevilli and their Antibacterial effects. Journal of Nanoscience and Nanotechnology 9(9): 5497-5501.

39. Suriya J, Bharathi Raja S, Sekar V, Rajasekaran R (2012) Biosynthesis of silver nanoparticles and its antibacterial activity using seaweed Urosporasp. African Journal of Biotechnology 11(58): 12192-12198.

40. Sangeetha N, Saravanan K (2014) Biogenic silver nanoparticles using marine seaweed (Ulvalactuca) and evaluation of its antibacterial activity. Journal of Nanoscience and Nanotechnology 2(1): 99-102.

41. Vijayan SR, Santhiyagu P, Ahila NK, Jayaraman R, Ethiraj K (2014) Synthesis and characterization of silver and gold nanoparticles using aqueous extract of seaweed, Turbinariaconoides and their Antimicrofouling activity. The Scientific World Journal.

42. Stalin Dhas T, Ganesh Kumar V, Karthick V, Jini Angel K, Govindaraju K (2014) Facile synthesis of silver chloride nanoparticles using marine alga and its antibacterial efficacy Spectrochimica Acta-Part A. Molecular and Biomolecular Spectroscopy 120: 416-420.

43. Romero-Gonz'alez E, Williams CJ, Gardiner PHE, Gurman SJ, Habesh S (2003) Spectroscopic studies of the biosorption of gold(III) by dealginated seaweed waste. Environmental Science and Technology 37(18): 4163-4169.

44. Chen X, Schluesener HJ (2008) Nanosilver: a nanoproduct in medical application. Toxicology Letters 176(1): 1-12.

45. Sotiriou GA, Pratsinis SE (2011) Engineering nano silver as an antibacterial, biosensor and bioimaging material. Current Opinion in Chemical Engineering 1(1): 3-10.
46. Luangpipat T, Beattie IR, Chisti Y, Haverkamp RG (2011) Gold nanoparticles produced in a microalga. Journal of Nanoparticle Research 13(12): 6439-6445.

47. Rajasulochana P, Dhamotharan R, Murugakoothan P, Murugesan S, Krishnamoorthy P (2010) Biosynthesis and characterization of gold nanoparticles using the alga Kappaphycus alvarezii. International Journal of Nanoscience 9(5): 511-516.

48. Senapati S, Syed A, Moeez S, Kumar A, Ahmad A (2012) Intracellular synthesis of gold nanoparticles using alga Tetraselmiskochinensis. Materials Letters 79: 116-118.

49. Hussain SM, Hess KL, Gearhart JM, Geiss KT, Schlager JJ (2005) In vitro toxicity of nanoparticles in BRL 3A rat liver cells. Toxicology in vitro 19(7): 975-983.

50. Arockiya Aarthi Rajathi R, Parthiban C, Ganesh Kumar V, Anantharaman P (2012) Biosynthesis of antibacterial gold nanoparticles using brown alga, Stoechospermummarginatum (k"utzing) SpectrochimicaActa-Part A. Molecular and Biomolecular Spectroscopy 99: 166-173.

51. Sharma B, Purkayastha DD, Hazra S, Lohit G, Chira RB, et al. (2014) Biosynthesis of gold nanoparticles using a freshwater green alga, Prasiolacrispa. Materials Letters 116: 94-97.

52. Liu X, Dai Q, Austin L, Janelle C, Genevieve K, et al. (2008) A one-step homogeneous immunoassay for cancer biomarker detection using gold nanoparticle probes coupled with dynamic light scattering. Journal of the American Chemical Society 130: 2780-2782.

53. Nagarajan S, Kuppusamy KA (2013) Extracellular synthesis of zinc oxide nanoparticle using seaweeds of Gulf of Mannar, India. Journal of Nanobiotechnology 11(39).

54. Abboud Y, Saffaj T, Chagraoui A, Brouzi K, Tanane O, et al, (2014) Biosynthesis, characterization and antimicrobial activity of copper oxide nanoparticles (CONPs) produced using brown alga extract (Bifurcariabifurcata). Applied Nanoscience 4(5): 571-576.

55. Khanehzaei H, Ahmad MB, Shameli K, Ajdari Z (2014) Synthesis and characterization of $\mathrm{Cu} @ \mathrm{Cu}_{2} \mathrm{O}$ core shell nanoparticles prepared in seaweed kappaphycus alvarezii media. International Journal of Electrochemical Science 9(12): 8189-8198.

56. Zhai T, Fang X, Liao M, Xijin X, Haibo Z, et al. (2009) A comprehensive review of one-dimensional metal-oxide nanostructure photodetectors. Sensors 9(8): 6504-6529.

57. Sudhagar P, Kumar RS, Jung JH, Cho W, Sathyamoorthy R, et al. (2011) Facile synthesis of highly branched jacks-like ZnO nano rods and their applications in dye-sensitized solar cells. Materials Research Bulletin 46(9): 1473-1479.

58. Cho S, Kim S, Jang JW, Seung HJ, Eugene 0, et al. (2009) Large-scale fabrication of sub- 20-nm-diameter $\mathrm{ZnO}$ nano rod arrays at room temperature and their photo catalytic activity. The Journal of Physical Chemistry C 113(24): 10452-10458.

59. Sapkota A, Anceno AJ, Baruah S, Shipin OV, Dutta J (2011) Zinc oxide nano rod mediated visible light photo inactivation of model microbes in water. Nanotechnology 22(21).

60. Van Horssen R, Ten Hagen TLM, Eggermont AMM (2006) TNF- $\alpha$ in cancer treatment: molecular insights, antitumor effects, and clinical utility. Oncologist 11(4): 397-408.

61. Zheng Y, Sanche L (2009) Gold nanoparticles enhance DNA damage induced by anti-cancer drugs and radiation. Radiation Research 172(1): 114-119.

62. Ahmadi SJ, Outokesh M, Hosseinpour M, Mousavand T (2011) A simple granulation technique for preparing high-porosity nano copper oxide (II) catalyst beads. Particuology 9(5): 480-485 